\title{
Culturing and transcriptome profiling of progenitor-like colonies derived from adult mouse pancreas
}

Dongshen Ma, Shanshan Tang, Jing Song, Qiong Wu, Fangfang Zhang, Yun Xing, Yi Pan, Yanfeng Zhang, Jingwei Jiang ${ }^{\dagger}$, Yubin Zhang ${ }^{\dagger}$ and Liang Jin $^{*+}$

\begin{abstract}
Background: Transplantation of insulin-producing cells is considered an important diabetes therapy. Many research studies have shown that insulin-producing cells can be derived from the in-vitro cultured pancreatic colonies with self-renewal ability and multilineage potential. Even though these progenitor-like colonies have been prepared from adult pancreas cells, the efficient culture method is hardly established and regulation of the colonies is rarely known. We confirmed previously that single cells acquired from adult mouse pancreas could form cyst-like colonies in a 3D semi-solid system containing Matrigel and methylcellulose. These colonies could be passaged continuously without losing progenitor-like capacity. In the previous culturing system, however, conditioned medium from murine embryonic-stem-cell-derived pancreatic-like cells was used. This unregulated ingredient may reduce repeatability and affect following study. Thus, a new culturing system with certain components needs to be developed.
\end{abstract}

Methods: Single cell suspension was acquired from adult mouse pancreas and cultured in a Matrigel-based 3D system with epidermal growth factor, Nicotinamide, B27, and Noggin to form ring colonies. Serial-passage assay was performed to evaluate self-renewal ability. Real-time polymerase chain reaction and immunostaining were used to detect the expression of progenitor-related genes. A 2D differentiation method was used to testify the multilineage potency of the colonies. High-throughput sequencing (HTS) of the colonies was performed to profile the differentially expressed genes.

Results: We developed a 3D culturing system deprived of conditioned medium to propagate those colonies with high proliferative efficiency. HTS of the transcriptome of mRNAs, microRNAs (miRNAs) and long noncoding RNAs (IncRNAs) showed differentially expressed genes compared to the whole pancreas (as control). In mRNAs, several surface marker genes were identified in the colonies. Moreover in noncoding RNAs, miR-21a, miR-31 and miR-155 were upregulated and miR-217, miR-802 and miR-375 were downregulated in colonies along with a number of other miRNAs and IncRNAs.

Conclusions: Our results offer an efficient culture system for pancreatic progenitor-like colonies and HTS of the colonies serves as a target resource for following study of in-vitro cultured pancreatic progenitors. These findings should also contribute to our understanding of the transcriptional regulation of these progenitor-like colonies and the mechanisms behind their functions.

Keywords: Pancreatic progenitors, 3D culturing system, High-throughput sequencing, MicroRNAs, Long noncoding RNAs

\footnotetext{
* Correspondence: ljstemcell@cpu.edu.cn

${ }^{\dagger}$ Equal contributors

State Key Laboratory of Natural Medicines, Jiangsu Key Laboratory of Drug

Screening, School of Life Science and Technology, China Pharmaceutical

University, 24 Tongjiaxiang, Nanjing, Jiangsu, People's Republic of China
} 


\section{Background}

Having been known for decades, adult pancreatic cells hold the promising prospect of being used for generating insulin-producing cells $[1,2]$. These progenitor-like cells were believed to be located in the duct, centroacina or acinar in various reports [3-5]. Among these, ductal epithelium is widely recognized as a potential pool in vitro and in vivo [6]. These cells remain quiescent in the adult, but when the pancreas is damaged or given certain substances they start to proliferate and then give rise to other pancreatic lineages including beta cells $[7$, 8]. These cells can differentiate into insulin-producing cells under specific conditions in vitro [9] or be directly transplanted under the kidney capsule for differention in-vivo [10]. Hence, an in-vitro culturing system serves as a pivotal tool to enrich them for transplantation.

Previously, we found that $\mathrm{CD} 133^{+} / \mathrm{SOX} 9^{\text {high }}$ cells in adult mouse pancreas could form cyst-like colonies in a 3D culture system [11]. These "ring" colonies maintain self-renewal capacity after several passages and can form endocrine/acinar colonies in laminin hydrogel. Given these facts, this system provides a good platform to research the regulation of the colonies' functions.

CD133 is a common marker for pancreatic ductal marker $[12,13]$. Sex determining region Y-box 9 (SOX9) is a transcriptional factor which plays an important role in pancreas development and is restricted mainly to the duct in adult mice [14]. Our previous study sorted out a $\mathrm{CD}_{133^{+}} / \mathrm{SOX}^{\text {high }}$ subpopulation to form progenitor-like colonies. However, this fraction may not represent all ductal epithelial cells which have colony-forming ability. Moreover, conditioned medium from murine embryonic stem cells has been applied in this culture system, containing uncontrollable components which may hinder the revealing of regulatory factors [11]. For the following analysis of the colonies' regulation, this prompted us to modify our previous culturing system by propagating single cells from the whole pancreas instead of interrogating only a subpopulation based on limited markers.

In our modified system, single cells from adult mouse pancreas can form $\mathrm{PDX}^{+}$colonies resembling the previous ones [13]. Pancreatic and duodenal homeobox 1 (PDX1) is a classic marker for pancreas specification and is expressed in ductal epithelium during development, which gives rise to all pancreatic lineages [15]. Ductoriginated progenitors derived from C57BL6 mouse regained the ability to express PDX1 if cultured in vitro, which could explain the pluripotency of these cultured progenitors $[11,13]$. During culture, PDX1 and proliferation marker Ki67 are expressed continuously in our colonies with stable ductal epithelium phenotype, indicating that they are proper materials for further investigation.

To accumulate more colonies for beta-cell generation or transplantation, the regulation of their functions (especially proliferation and differentiation) should be examined carefully to find vital regulators. Therefore, we performed high-throughput sequencing (HTS) of the transcriptome of the colonies versus whole pancreas to find specific RNAs in the colonies. HTS, also named "next generation sequencing", is extensively used to profile RNA transcriptome in cancer stem cells [16] and adult stem cells [17]. With this technology, coding mRNAs and noncoding RNAs (microRNAs (miRNAs) and long noncoding RNAs (lncRNAs)) can be quantified accurately and efficiently, making it a great help in development and stem/progenitor cell study [18].

In addition to illustrating the properties of specific cells, HTS can also provide a large amount of valuable information such as new surface markers. Surface markers are surface antigens located within the cell membrane. They can be detected easily by flow cytometry (FCM), making the cells sortable without gene modification [19]. Discovery of new surface markers and sorting for new subpopulations of pancreatic progenitor-like colonies can help the enrichment of potential cells with high colony-forming efficiency. Some of the specific markers have been discovered, including our previously reported CD71 [20]. In this study we recorded 7266 mRNA changes in our colonies compared to pancreas. By analyzing mRNAs, we captured significant changes in gene expressing profile including surface antigens which could be used as surface markers for cell sorting.

Along with mRNAs, 285 miRNA and 183 lncRNA changes were detected. miRNAs are small noncoding RNAs which can bind target mRNAs to silence their expressions [21]. miRNAs have critical effects on the pancreatic differentiation from pluripotent stem cells [22] and beta-cell functions [23]. Our previous study has shown that miR-26a is regulated in differentiation by targeting ten-eleven translocation (TET) enzymes [24]. lncRNAs are also noncoding RNAs discovered recently. They regulate gene expression through many mechanisms [25]. There have been plenty of studies on lncRNAs regulating pancreatic cancer [26], beta-cell biology [27] and pancreas development [28]. However, neither miRNAs nor lncRNAs are deeply researched in regulation of pancreatic progenitor-like colonies.

Our study for the first time profiled the transcriptome of pancreatic progenitor-like colonies. Compared to transcriptome of whole pancreas, we found highly upregulated mRNAs, miRNAs and IncRNAs along with potential surface markers. With Gene Ontology (GO) and Kyoto Encyclopedia of Genes and Genomes (KEGG) analysis, these findings should help the understanding of these colonies and the regulatory network of proliferation and differentiation. 


\section{Methods}

\section{Animals}

C57BL/6 J mice (6-8 weeks old) were obtained from the Model Animal Research Center of Nanjing University (Nanjing, China). All care and handling of animals were carried out according to the international laws and policies (EEC Council Directive 86/609, 1987) and approved by the animal ethics committee of China Pharmaceutical University (Nanjing, China) (Reference Number: 2162326).

\section{Single cell preparation and culturing}

For 3D culture, pancreas from 8-week-old C57BL/6 J mouse was isolated and cut into $1-\mathrm{mm}^{3}$ pieces on a cold dish, followed by 30-min digestion in $2 \mathrm{mg} / \mathrm{ml} \mathrm{col-}$ lagenase II at $37{ }^{\circ} \mathrm{C}$ to obtain single cells. Then 8000 live cells were counted after trypan blue staining and were seeded into a 24-well ultra-low attachment plate (Corning) in DMEM/F12 (Gibco) supplied with 10\% Matrigel (Corning), 1\% methylcellulose (1500 cP; Sigma), $20 \mathrm{ng} / \mathrm{ml}$ EGF (Sigma), $10 \mathrm{mM}$ Nicotinamide (Sigma), $100 \mathrm{ng} / \mathrm{ml}$ Noggin, 2\% B27 (Invitrogen), 5\% FBS (Gibco), $100 \mathrm{U} / \mathrm{ml}$ penicillin and $100 \mu \mathrm{g} / \mathrm{ml}$ streptomycin (Gibco).

For 2D culture, ring colonies were dissolved in $0.25 \%$ trypsin to obtain single cells. Cells were counted and seeded in Matrigel-coated (10\%) plates in DMEM/F12 supplied with $5 \mu \mathrm{M}$ SB431542 (Sigma), $20 \mathrm{ng} / \mathrm{ml}$ EGF, $10 \mathrm{mM}$ Nicotinamide (Sigma), 1\% B27, 5\% fetal bovine serum (FBS), $100 \mathrm{U} / \mathrm{ml}$ penicillin and $100 \mu \mathrm{g} / \mathrm{ml}$ streptomycin (Gibco).

For beta-lineage differentiation, the method reported previously was employed [29]. Cells were cultured in a 2D system with serum-free DMEM/F12 supplemented with ITS $(5 \mathrm{mg} / \mathrm{l}$ insulin, $5 \mathrm{mg} / \mathrm{l}$ transferrin. $5 \mu \mathrm{g} / \mathrm{l} \mathrm{so-}$ dium selenite; Gibco), 2 g/l bovine serum albumin (BSA; Biosharp), $8 \mathrm{mmol} / \mathrm{l}$ glucose (Sigma), $10 \mathrm{mmol} / \mathrm{l} \mathrm{nico-}$ tinamide, $100 \mathrm{U} / \mathrm{ml}$ penicillin and $100 \mu \mathrm{g} / \mathrm{ml}$ streptomycin (Gibco). After the medium was changed, 10\% Matrigel was applied on the top of the cells with overnight gelling at $37^{\circ} \mathrm{C}$.

Colonies were cultured in $37{ }^{\circ} \mathrm{C}$ with $5 \% \mathrm{CO}_{2}$ for $2-4$ weeks depending on the experiments.

\section{Serial passaging assay}

For 3D culture, after 2 weeks of culture all of the colonies were counted and hand-picked under an inverted microscope. After dissolving in $0.25 \%$ trypsin, cells were counted and replated in the same condition. This process was repeated five times.

\section{RNA extraction}

For the pancreas, adult mice were sacrificed and dissected immediately to isolate the pancreas. Fresh pancreas was cut into $3-\mathrm{mm}^{3}$ pieces and immersed in RNAlater (Qiagen) for $1 \mathrm{~h}$ at room temperature before extraction. For ring colonies, single colonies were handpicked using a $20-\mu \mathrm{l}$ pipette under an inverted microscope and collected in a 1.5-ml tube. For adherent cells, QiAzol Lysis Reagent (Qiagen) was directly added to the cells to obtain cell lysate. All samples were then homogenated and RNA was extracted using the Qiagen RNeasy Mini kit (Qiagen).

\section{Real-time PCR and RT-PCR}

The real-time PCR and RT-PCR were performed following standard procedures. CDNA was prepared using either All-In-One RT MasterMix (ABM) or the specific miRNA/lncRNA reverse transcription kit (Genepharma). Real-time PCR was performed using EvaGreen 2X qPCR MasterMix (ABM) on a Light Cycler 480 II (Roche). RT-PCR was performed using the EasyScript Two-Step RT-PCR Kit (ABM) on a normal PCR instrument (Thermo). The RT-PCR products were separated and detected using $1.5 \%$ agarose (Lonza). The primers used in RT-PCR and real-time PCR are presented in Additional file 1: Table S1.

\section{Immunostaining}

Colonies were hand-picked using a 20- $\mu$ l pipette, fixed in $4 \%$ paraformaldehyde (Sigma) for $10 \mathrm{~min}$ at room temperature and blocked with Blocking Buffer (Abcam) at $37{ }^{\circ} \mathrm{C}$ for $1 \mathrm{~h}$, followed by primary antibody incubation with PDX1 (1:500), Ki67 (1:200), cytokeratin 7 (CK7; 1:250), neurogenin 3 (NEUROG3; 1:50), SOX9 (1:200), or C-peptide (1:250). The colonies were finally detected with secondary antibodies conjugated to Alexaflour 488 or Alexaflour 650 (Abcam) at a dilution of 1:250. Images were captured by a laser scanning confocal microscopy (Olympus) or an inverted fluorescence microscope (Olympus).

\section{Flow cytometry and cell sorting}

For the pancreas, single cells were prepared as already mentioned. For colonies, single colonies were handpicked and digested in trypsin. Cells were blocked using CD16/32 antibody (eBioscience) and stained with CD133-APC antibody (eBioscience) or its isotype antiRat IgG1 (eBioscience). After washing twice in PBS, the $\mathrm{CD}_{133^{+}}$cells were detected by Accuri C6 (BD) or sorted by Aria (BD).

\section{High-throughput sequencing}

Three independent samples of colonies and their preculture controls (pancreas) were harvested for HTS. RNA was extracted as already mentioned. An RNA library was constructed using the TruSeq RNA LT Sample Prep Kit. Then the cluster generation process was performed 
using the TruSeq PE Cluster Kit on a cBot system. The sequencing process was performed using the TruSeq SBS Kit on a Hiseq2500. All of the kits and instruments were obtained from Illumina. Also, Newbler2.7 was used for data analysis and Mus musculus GRCm38.p5 (Ensemble) was used for comparison.

\section{Statistical analysis}

Data are presented as the mean \pm SD. Two-tailed $t$ test was used to assess the differences between experimental groups. Statistical significance was defined as $p<0.05, p$ $<0.01$ and $p<0.005$.

\section{Results}

Dissociated mice pancreas forms cyst-like ring colonies in modified 3D medium

To replace the conditioned medium from murine embryonic stem cells used in our previous 3D culture system, new ingredients were applied and the concentration of Matrigel was elevated to $10 \%$. After culturing for 2 weeks (Additional File 2: Figure S1A), organoidlike ring colonies formed in concentrated Matrigel (Fig. 1a) with a colony-forming efficiency of about $1.2 \%$ (Additional file 3: Table S2), resembling the published data [13]. The diameters of most colonies were under $500 \mu \mathrm{m}$ (Additional File 2: Figure S1B). The cell

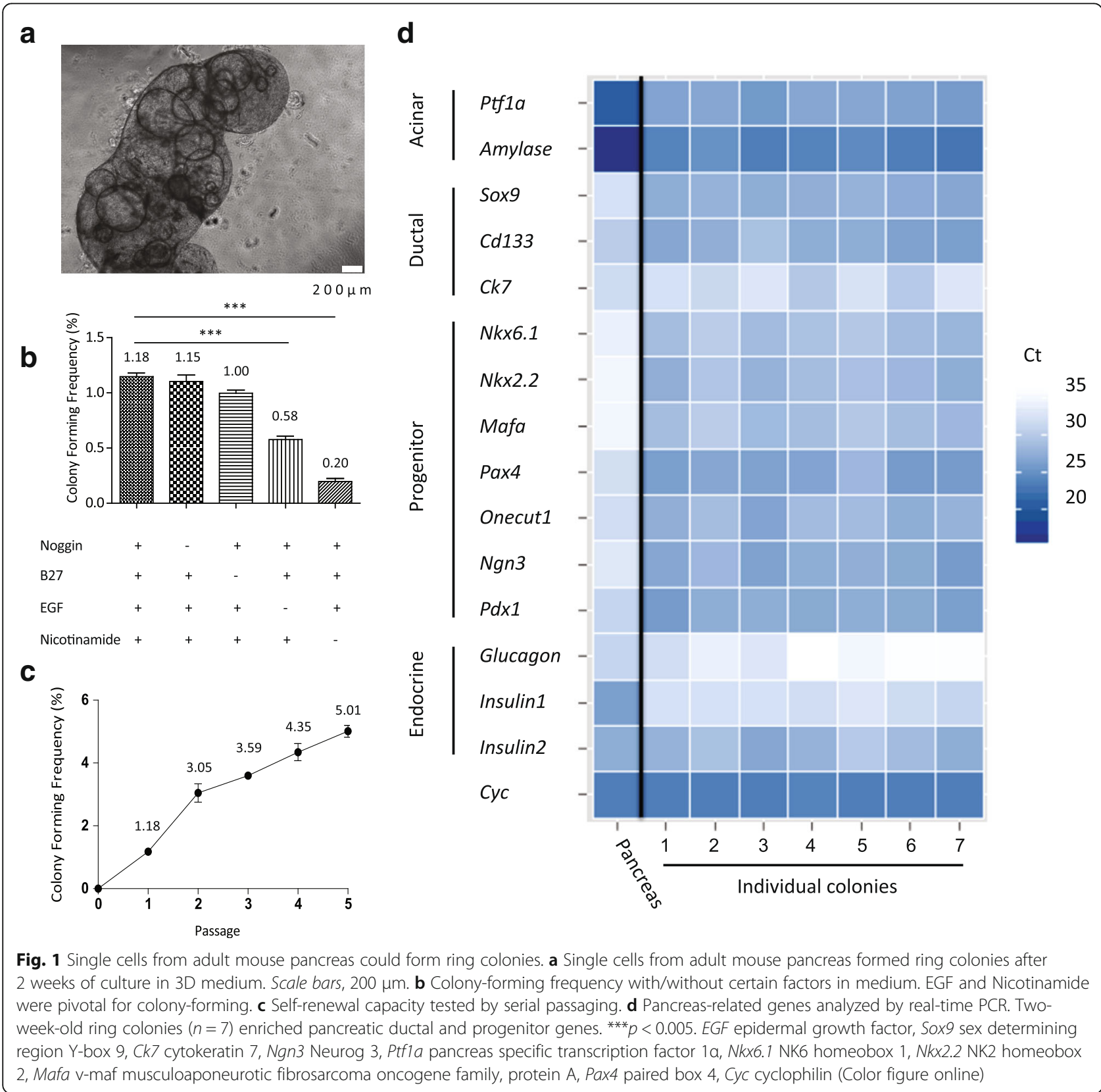


numbers of different colonies are represented in Additional File 2: Figure S1C with a fitted curve.

We knocked out each of the components in our medium (except FBS) to interrogate its impacts on colony-forming frequency. Of note, EGF and Nicotinamide are pivotal for colony-forming while Noggin and B27 exhibited slight effects (Fig. 1b). Also, DMEM/F12 showed more efficiency than H-DMEM and L-DMEM (Additional File 2: Figure S1D).

Self-renewal is one of the properties of pancreatic progenitors and allows them to be passaged continuously in vitro. Thus, a serial passaging method was performed to evaluate the self-renewal ability of our colonies. Twoweek-old colonies were hand-picked and trypsinized. The cells were then replated in new medium until secondary ring colonies emerged. As shown in Fig. 1c, the colony-forming frequency increased along with the cell passage but the colony size was smaller after subculture for five passages (Additional File 2: Figure S1E). The results indicated that colony-forming cells were enriched in this culture system and they did not lose the capacity of self-renewal when subcultured at least within five passages, while somehow its proliferation ability declined.

To assess the mRNA expression pattern of the colonies, we used real-time PCR to test a sum of pancreatic specific genes or those relating to pancreas development. Two-week-old ring colonies were picked individually and dissolved for RNA extraction. Nearly all of the pancreatic specific genes were detected in ring colonies (Fig. 1d). Compared to pancreas, the colonies highly expressed ductal marker Sox9, CD133, Ck7 and other endocrine-relevant genes in pancreas development such as NK6 homeobox 1 (Nkx6.1), NK2 homeobox 2 (Nkx2.2), paired box 4 (Pax4), Neurog3, etc. Detected acinar and mature endocrine transcripts in the colony indicated pluripotency when cultured in vitro.

\section{Ring colonies express pancreatic progenitor markers confirmed by immunostaining}

Transcriptional factors which are critical in pancreas development such as PDX1 reoccur in cultured pancreatic progenitors [8]. Our data showed extensive PDX1 expression in ring colonies along with proliferation marker Ki67 (Fig. 2a). Expression of CK7 and SOX9 confirmed its ductal origin (Fig. 2b, c). Endocrine progenitor marker NEUROG3 was also detected (Fig. 2d). Although weak, the presence of NEUROG3 suggested the potential to generate endocrine lineages. Interestingly, after 3 weeks of culture, some of the colonies $(\sim 10 \%$, especially in large colonies) became shriveled and compact (Additional File 4: Figure S2). C-peptide, a marker for de-novo synthesized insulin, was detected in the cytoplasm (Additional File 4: Figure S2), demonstrating obvious endocrine commitment. These results indicated that, in our modified 3D culture system, ring colonies showed a progenitor-like phenotype and gene expression pattern. Moreover, with Ki67 expressed widely, our system offered a proper environment for proliferation.

\section{Cells in the colonies can be expanded as 2D plaques and form C-peptide secreting clusters}

To further assess the multilineage potency of our cultured colonies, we applied a method published previously to induce pancreatic progenitors to beta lineage [29]. Before differentiation, the cells in our colonies have to be plated into the $2 \mathrm{D}$ system. We employed $2 \mathrm{D}$ medium used to culture embryo stem cell-derived pancreatic progenitors (Fig. 3a). As expected, after dissociation from ring colonies and seeding in a Matrigelcoated plate, the cells (after three passages in a 3D system as colonies) formed epithelial plaques in 24-48 h (Fig. 3b). After 5 days of culture, cells were grown into a confluent monolayer (Fig. $3 \mathrm{~b}$ ). We passaged the cells in a 1:2-1:3 ratio every 5 days at least five times without an observed shape change (data not shown).

Confirmed by immunostaining, 2D plaques recapitulated the expression of CK7, PDX1 and Ki67 in ring colonies (Fig. 3c). The differentiation method was applied to induce the colony-derived cells to beta lineage after the plaques reached $100 \%$ confluence. Most of the cells went through apoptosis after being plated over 1 week, with NEUROG3 being broadly detected (Fig. 3d). Two weeks later, small dense clusters appeared (Fig. 3e) at a frequency of about 1.5 colonies per 10,000 cells. The presence of insulin in the clusters was confirmed by Dithizone (DTZ) staining (Fig. 3f). C-peptide was detected, indicating the forming of beta-cell-like clusters (Fig. $3 \mathrm{~g}$ ). Besides, exocrine genes Amylase, Somatostatin and chymotrypsin-like elastase (Cela1) and endocrine genes Insulin1 and Insulin2 were confirmed to be expressed in some of the clusters after induction (Additional File 5: Figure S3), which signified the pluripotency of the cells in the colonies.

\section{High-throughput sequencing and transcriptome profiling of the colonies}

We performed HTS to illuminate the gene expression pattern of our colonies and to discover the regulation factors. RNA of hand-picked colonies and whole pancreas (as control) was extracted, purified and sequenced. Compared to the pancreas, 7266 mRNAs, 285 miRNAs (111 previously unidentified) and 183 lncRNAs (23 previously unidentified) were found differentially expressed in colonies (Fig. 4a, Additional File 6: Figure S4A and Additional File 7: Table S3). The top 25 differentially expressed mRNAs, miRNAs and lcnRNAs were listed (Additional file 8: Table S4, Additional file 9: Table S5 


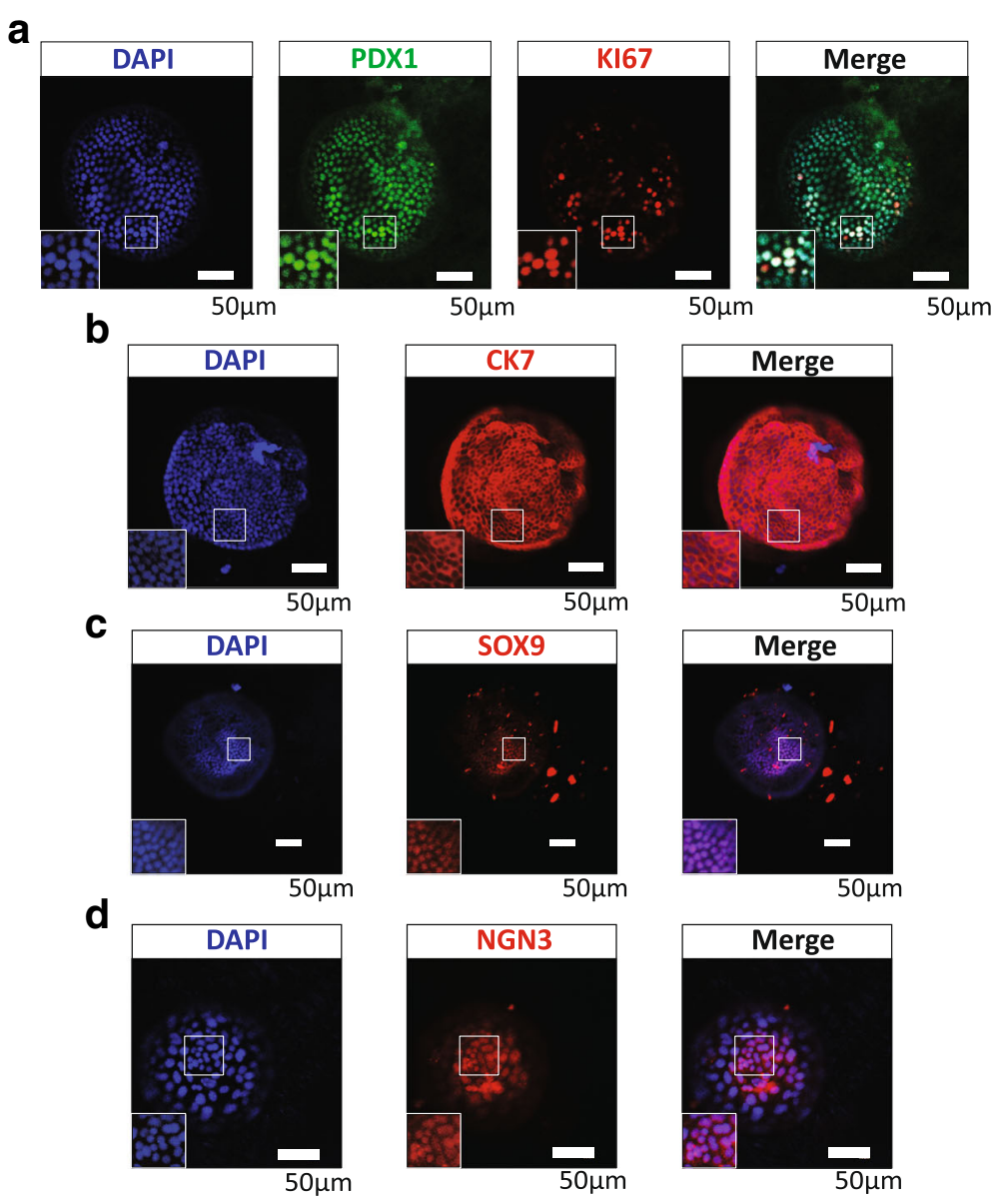

Fig. 2 Immunostaining of pancreatic progenitor-related transcriptional factors in ring colonies. a-d Whole-mount immunostaining of single ring colonies showed PDX1/Ki67, CK7, SOX9 and NEUROG3 expression. Scale bars, $50 \mu \mathrm{m}$. PDX1/Ki67 coexpression indicated a proliferative pancreatic progenitor-like phenotype. CK7 and SOX9 showed the ductal origin of the colony. Weak expression of NEUROG3 implied its potential for endocrine lineage. SOX9 sex determining region Y-box 9, CK7 cytokeratin 7, NGN3 neurog 3, PDX1 Pancreatic and duodenal homeobox 1 (Color figure online)

and Additional file 10: Table S6). The original datasets for HTS are also provided (Additional file 11: Table S7 and Additional file 12: Table S8).

Surprisingly, in upregulated mRNAs, it was not the classical pancreatic progenitor-related genes that changed the most. Methyltransferase like 10 (Mettl10), a methyltransferase-like gene, took first place of the upregulated genes. Although not functionally characterized, it has been found to specifically trimethylate eukaryotic translation elongation factor 1 alpha 1 (Ef1a1), a eukaryotic elongation factor, which implies its contribution to many biological processes on translational level [30]. Onecut2, a member of the onecut family, is also among those most upregulated genes. It is known as downstream of paired box 6 ( $P a x 6)$, and least partially contributed to pancreas specification and endocrine differentiation [31, 32]. Another upregulated gene, fibroblast growth factor receptor 2 ( $F g h r 2)$, indicated the important role of FGF signal in regulating of the colonies. Inhibition of $F g h r 2$ in pancreatic cancer has been shown to impair proliferation [33], implying its proliferation-promoting function on pancreas-originated cells. On the other hand, pancreatic exocrine genes such as trypsin 4 (Try4), chymotrypsin (Ctrl) and Insulin1 were among the downregulated genes, indicating the expected differential between colonies and pancreas control.

We focused on some of the transmembrane protein genes of significantly changed mRNAs. These up/downregulated genes could be regarded as a positive/negative surface marker pool for screening. We verified 10 of these genes by real-time PCR (Fig. 4b). Some signal pathway receptors (delta-like 1 (Dll1), frizzled class receptor $1(F z d)$ ), some cell adhesion molecules (intercellular adhesion molecule 1 (Icam1) and other clusters of differentiation (CDs) were among them. Especially, CD133, a classic marker for pancreatic stem cells [19], was upregulated in our colonies ( 4-fold higher in HTS results). 


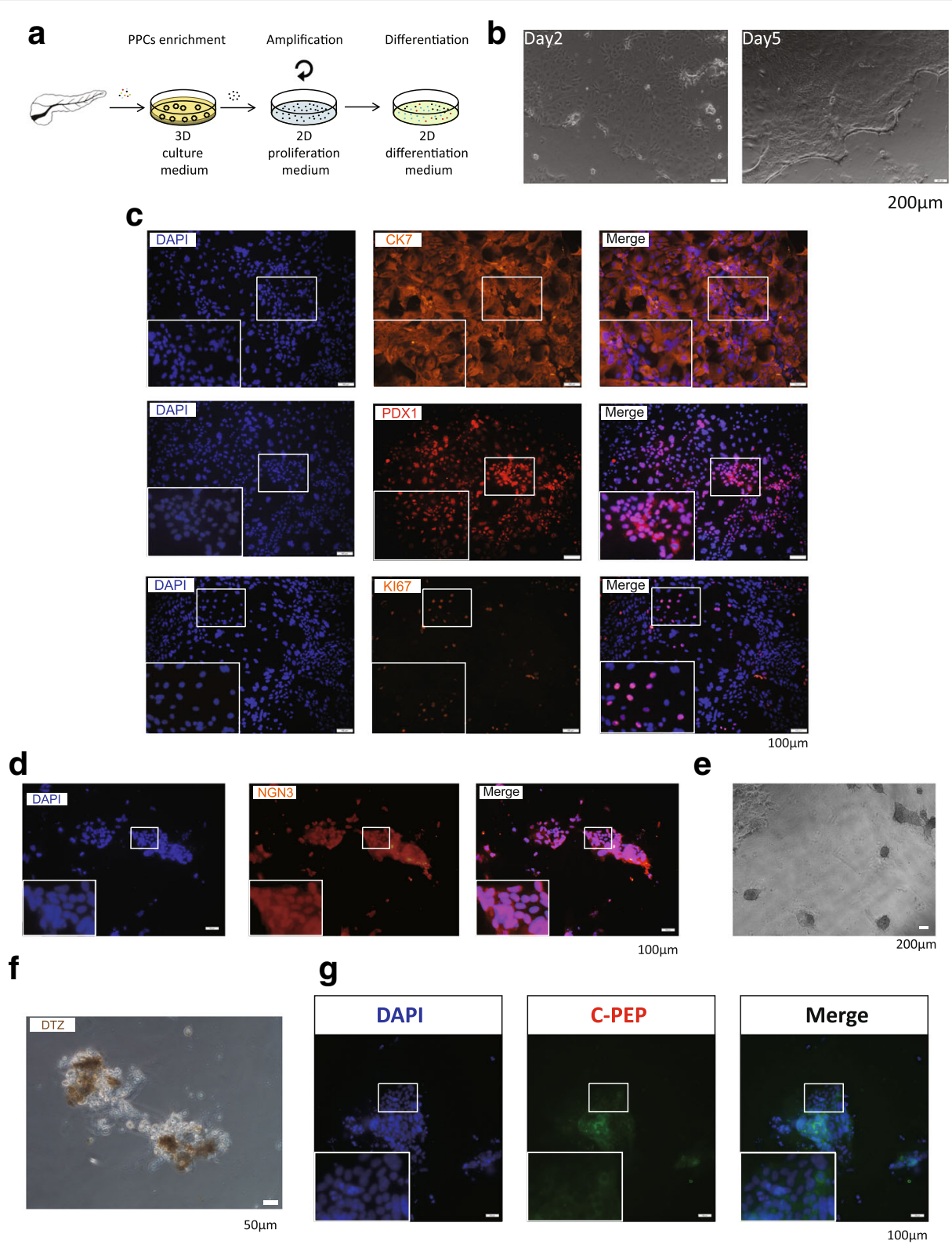

Fig. 3 Cells from ring colonies can be induced to differentiate to beta lineage. a After enrichment in a 3D system, colonies after three passages were dissolved and plated in a 2D system for differentiation. Scale bars, $200 \mu \mathrm{m}$. b Plaques formed in a 2D system and cells reached nearly 100\% confluence in 5 days. c Immunostaining showing expression of CK7, PDX1 and Ki67 in 2D plaques. Scale bars, 100 um. d NEUROG3 detected after 1 week of culture in differentiation medium, indicating the commitment of endocrine progenitors. Scale bars, $200 \mu \mathrm{mm}$. e- $\mathbf{g}$ Clusters found at day 14 in differentiation medium and the staining of DTZ and C-peptide was positive. Scale bars, $50 \mu \mathrm{m}$. C-pep C-peptide, CK7 cytokeratin 7, DTZ Dithizone, PDX1 Pancreatic and duodenal homeobox 1 (Color figure online)

We also verified eight miRNAs (Fig. 4c). We noted that miR-31-5p, miR-21a-3p/5p, miR-155-5p and miR$29 \mathrm{~b}-3 \mathrm{p}$ were upregulated. miR-31 is engaged in regulating the proliferation of pancreatic cancer cells [34], indicating its potential role in regulating pancreaticoriginated cells. miR-21a has been reported recently to be an important regulator in pancreatic progenitors in chicken [35]. miR-155 and miR-29b were recently reported upregulated in the pancreas of a transgenetic model mouse for diabetes [36]. In downregulated miRNAs, miR-216a-3p/5p, miR-216b-3p/5p, miR-217$5 p, m i R-802-3 p / 5 p$ and miR-375-3p lay on the top. miR-216/miR-217 were supposed to be reduced in pancreatic adenocarcinoma [37]. miR-802 is highly expressed in the liver and pancreas, and regulates insulin resistance by targeting HNF1 homeobox B (HNF1B) in the liver [38], while its function in the pancreas remains unknown. miR-375 is a well-known 


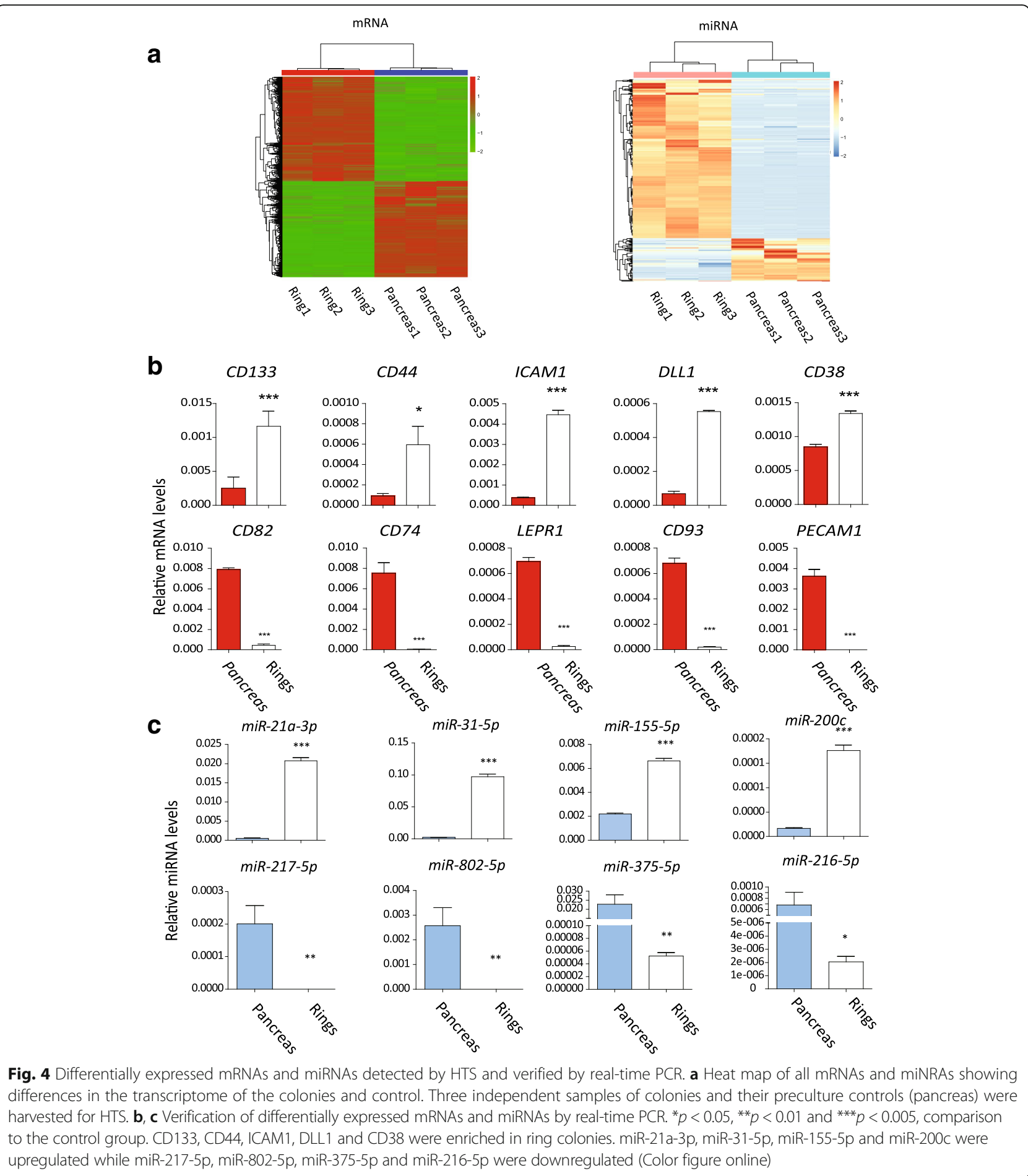

miRNA in regulating beta-cell development [39] and is used to generating insulin-producing cells from induced pluripotent cells [40]. The reported function of these mostly changed miRNAs is summarized in Table 1. However, none of these miRNAs is deeply studied in pancreatic progenitors. Moreover, eight
lncRNAs were also verified (Additional File 6: Figure S4B), including metastasis associated lung adenocarcinoma transcript 1 (Malat1), a lncRNA recently shown to promote pancreatic cancer cell proliferation [41]. Little is known about these lncRNAs in pancreas development or functions. 
Table 1 Reported functions of the most significantly changed miRNAs

\begin{tabular}{lllll}
\hline miRNA & Up/downregulated & Fold change $\left(\log _{2}\right)$ & Function & Reference \\
\hline miR-31-5p & Upregulated & 10.19 & Regulating migration of PDAC & [34] \\
miR-21a-5p & Upregulated & 7.89 & Regulating pancreatic progenitors in chicken & a \\
miR-155-5p & Upregulated & 7.80 & Biomarker in PDAC & [35] \\
miR-217-5p & Downregulated & -9.10 & Reduced in mouse model of PDAC & [36] \\
miR-802-5p & Downregulated & -6.81 & Targeting HNF1B & [37] \\
miR-375-3p & Downregulated & -6.66 & Promotes beta pancreatic differentiation in hiPS & [38]
\end{tabular}

PDAC pancreatic ductal adenocarcinoma, hiPS human induced pluripotent stem cells

These miRNAs were among the most significantly changed. They have been reported to be important in regulation of either pancreatic progenitors or pancreatic ductal adenocarcinoma

ammu-miR-21a-5p is homologous to gga-miR-21 in chicken

bPrevious ID of mmu-miR-375-3p is "mmu-miR-375"

\section{Colonies from whole pancreas are comparable to $\mathrm{CD} 133^{+}$ cell-derived colonies}

CD133 is a well-recognized marker for pancreatic progenitors $[11,12,19]$. HTS results and real-time PCR showed higher expression of CD133 in colonies (Fig. 4b). We then tested the CD133 expression in colonies using FCM. As shown in Fig. 5a, only $~ 4 \%$ cells were $\mathrm{CD} 133^{+}$while in the colonies more than $90 \%$ of the cells were $\mathrm{CD} 133^{+}$. $\mathrm{CD} 133^{+}$and $\mathrm{CD} 133^{-}$cells were sorted respectively through $\mathrm{BD}$ Aria and seeded in a 3D system. Surprisingly, only $\mathrm{CD}_{133^{+}}$cells could form "ring" colonies in our system after 2 weeks (Fig. 5b). Real-time PCR confirmed that $\mathrm{CD} 133^{+}$cell-derived colonies shared the same gene expression pattern with the colonies from the total pancreas cells (Fig. 5c). These results indicated that the ring colonies should be derived from the $\mathrm{CD}_{133^{+}}$subset of pancreatic cells and could be reliable materials for HTS because all colonyforming subsets were included.

\section{GO and KEGG enrichment of target genes}

GO is a dataset to annotate and classify all differentially expressed genes in order to reveal the most changed components or functions. Each GO category includes many terms (listed by gene counts) into which differentially expressed genes (in this study, colonies versus control) distribute. For noncoding RNA, GO analysis is applied to its target genes. The $p$ value is calculated for each GO term to represent the probability that gene counts in such a term could result from random distribution.

The top 10 GO terms of differentially expressed genes in the colonies were listed by $p$ value (Fig. 6a). In mRNA, differentially expressed genes were enriched in the biological processes of "cellular metabolic progress", "metabolic process" and "primary metabolic process". The cellular component genes were mainly enriched in "intracellular", "intracellular parts" and "cytoplasm". The molecular functions of these genes were "binding", "catalytic activity" and "protein binding". For predicted miRNA targets, differentially expressed genes were enriched in the biological processes of "metabolic process", "organic substance metabolic process" and "primary metabolic process". The cellular component genes were mainly enriched in "intracellular", "intracellular part" and "organelle". The molecular functions of these genes were "binding", "protein binding" and "catalytic activity". For lncRNA target genes, the top GO terms were also listed (Additional File 13: Figure S5A).

KEGG analysis showed the pathways in which differentially expressed genes were enriched. KEGG analysis of the mRNA transcriptome showed that "Ribosome" and "Metabolic pathways" were the most enriched pathways (Fig. 6b, upper). In miRNA target genes, "Pathways in cancer", "PI3K-Akt signaling pathways", "MAPK pathways" and "Focal adhesion" were the most enriched pathways (Fig. 6b, lower). Pathways enriched in lncRNA target genes are shown in Additional File 13: Figure S5B.

\section{Coexpression analysis of differentially expressed RNAs}

One of the mechanisms of miRNAs in regulating mRNAs is through the cleavage of mRNA [21], indicating that the expression trend of a miRNA and its target mRNA is likely to be opposite. Also, a lncRNA can affect the expression of mRNAs through specific mechanisms [25]. Thus the overlapped genes (targets) among significantly changed mRNAs, miRNAs and lncRNAs may play important roles in regulating the network.

To find key mRNAs regulated by the noncoding RNA network, we performed coexpression analysis of differentially expressed RNAs between the colonies and control. The intersection of significantly changed mRNAs and the potential targets of all significantly changed noncoding RNAs were obtained (Fig. 7a). We noted 304 overlapped genes. The top five up/downregulated genes were listed (Additional file 14: Table S9). The most upregulated genes were Cttnbp2, Zfp608, SorbS2, 


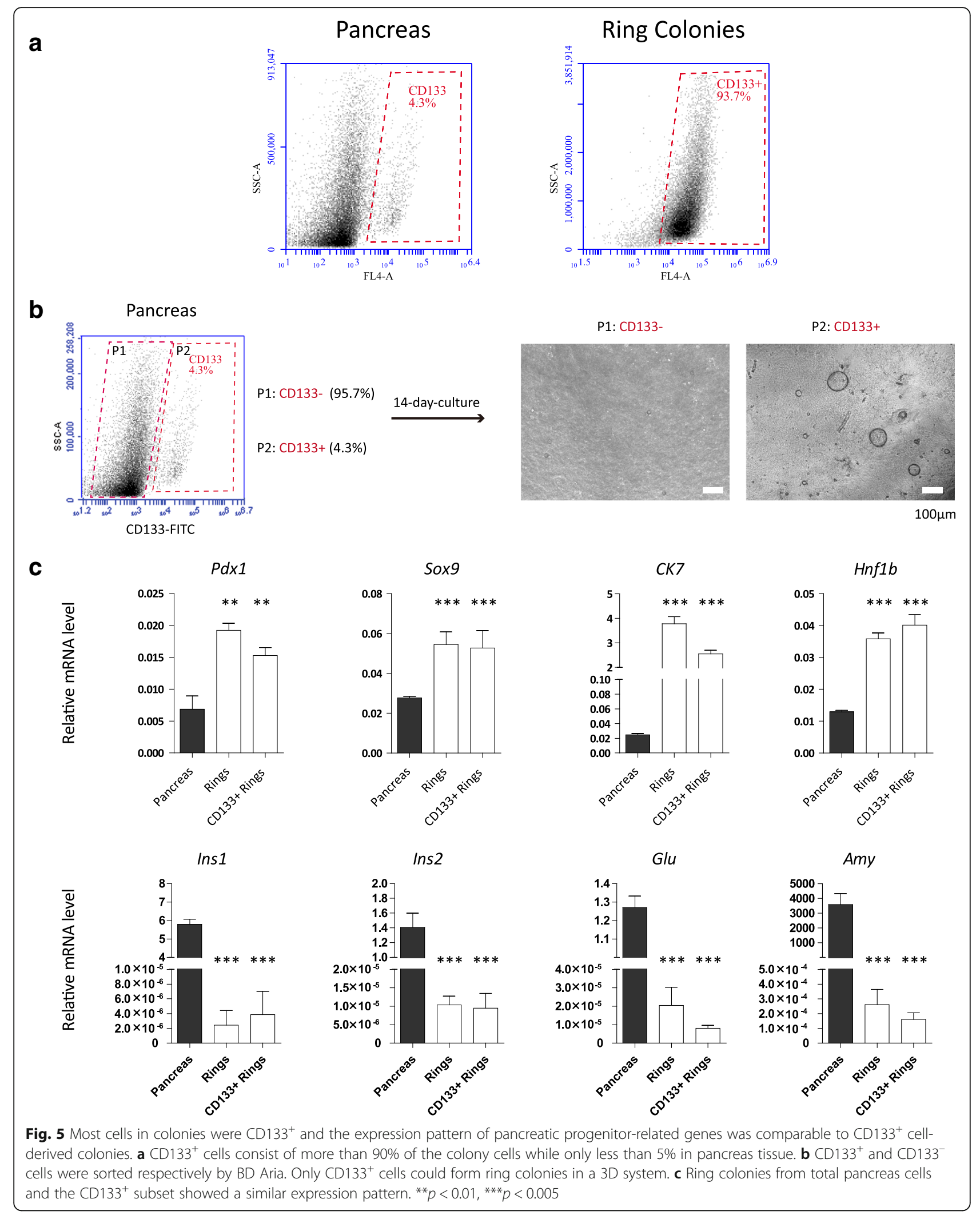



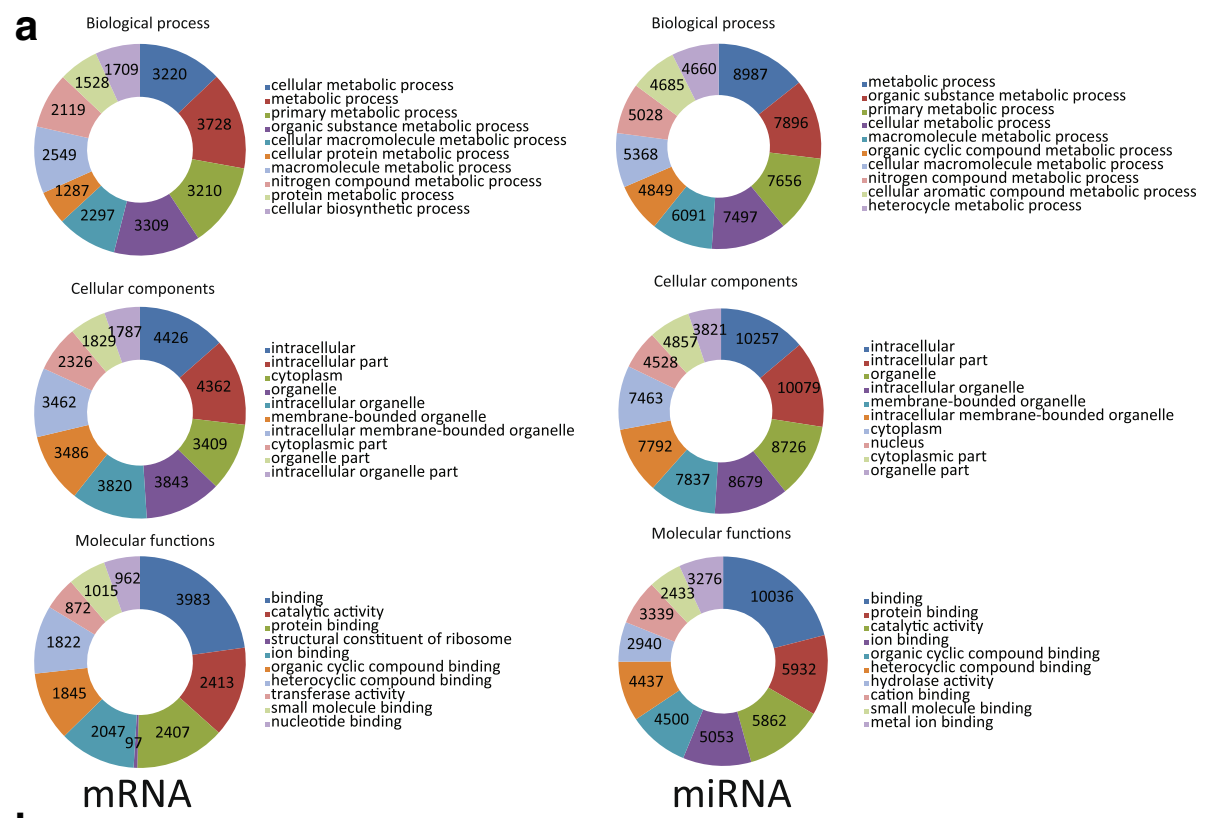

b
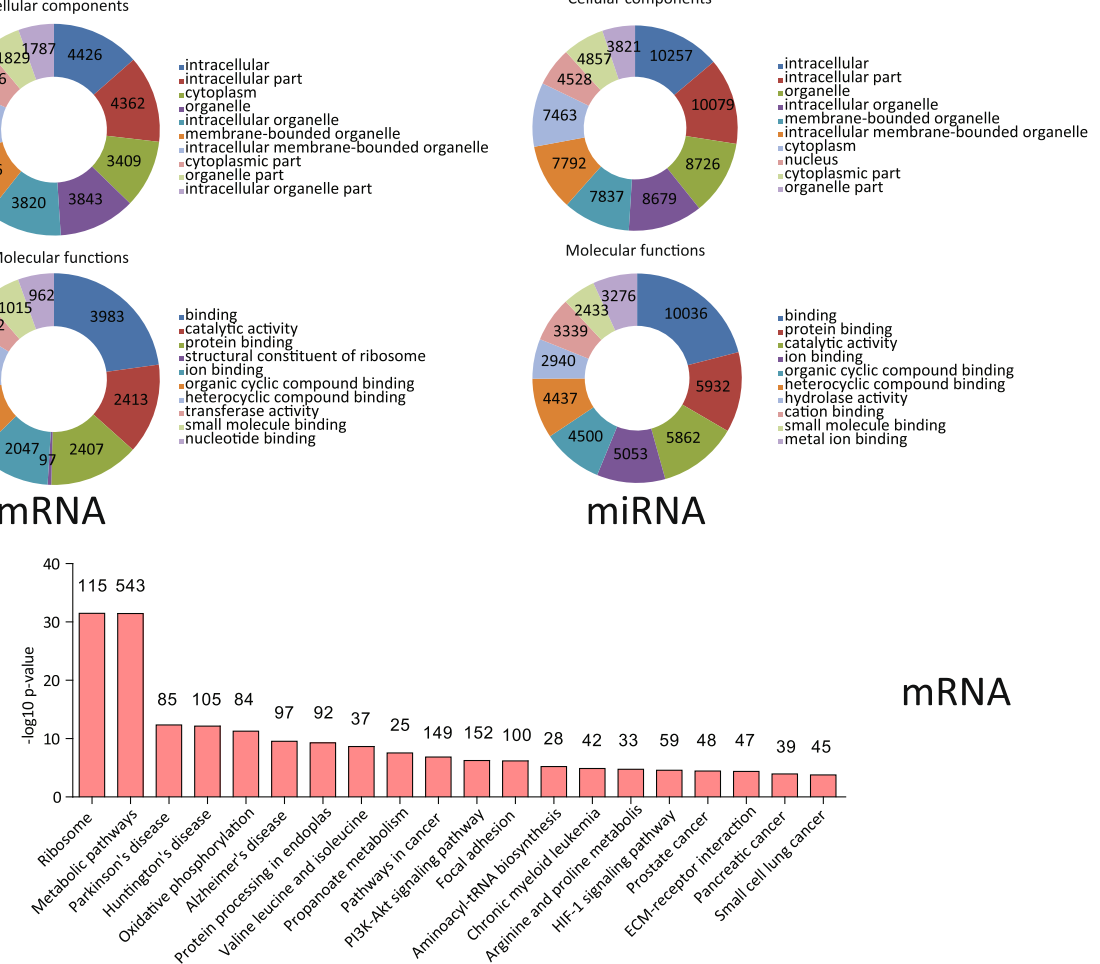

mRNA

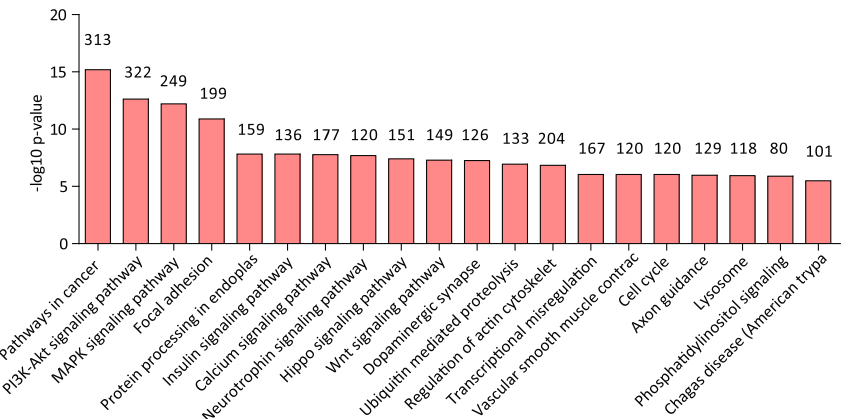

miRNA

Fig. $6 \mathrm{GO}$ and KEGG enrichment of mRNAs and miRNAs. a GO enrichment of significantly changed mRNAs (left) and miRNAs (right). Top 10 (by $p$ value) for each $\mathrm{GO}$ dataset shown. Numbers in each part of the graph stand for the gene counts in each GO dataset. b Top 20 (by $p$ value) of enriched KEGG pathways of mRNAs and miRNAs. Numbers on top of each column stand for the gene counts in each KEGG pathway. miRNA microRNA

Fam227a and St3gal. The most downregulated genes were Cd163, Padi2, Cth, Hsbp1l1 and Nphs1. Moreover, the GO and KEGG pathways were analyzed (Fig. 7b, c). Genes were enriched in the biological process of "metabolic process", the cellular components were enriched in "intracellular organelle" and the molecular functions were enriched in "ion binding". The most enriched KEGG process was "pyrimidine metabolism".

\section{Discussion}

Loss of Langerhans' islet occurs in both type I and type II diabetes [42, 43]. Currently, islet transplantation is the main way to cure diabetes completely. However, the lack of available donor islets has prevented extensive use of this method [44]. Thus, identification of alternative islet sources may provide a gateway to widespread use of this practice to treat diabetes. 
a

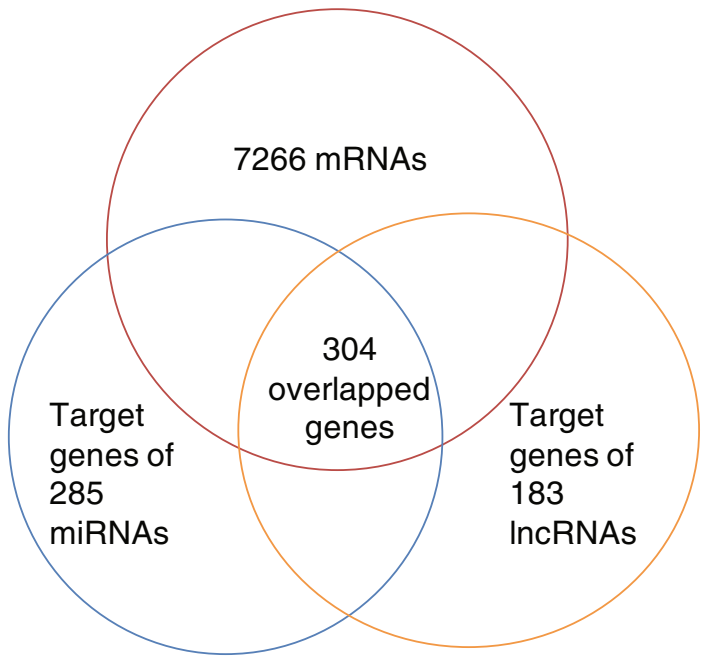

b

GO enrichment

Molecular functions

Cellular components

Biological pathways

Histone binding ransporter activity Catalytic activity Oxidoreductase activity, acting on the $\mathrm{CH}-\mathrm{NH} 2$ group. TBP-class protein binding Coenzyme transporter activity
Primary amine oxdiase activity Primary amine oxdiase activity
Oxidoreductase acticity, oxygen as acceptor. lon binding. Cytoplasm. Mitochondrial part Mitochondrial matrix Mitochondrion Mitochondrion.
Intracellular. Intracellular.
Intracellular part. Membrane-bounded organelle Intracellular membrane-bounded organelle Intracellular organelle Catecholamine catabolic process Pyrimidine deoxyribonucleotide biosynthetic process Diol catabolic process Coenzyme transport Gene expression

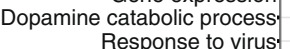
Defense response to virus Cellular amino acid metabolic process Metabolic process.

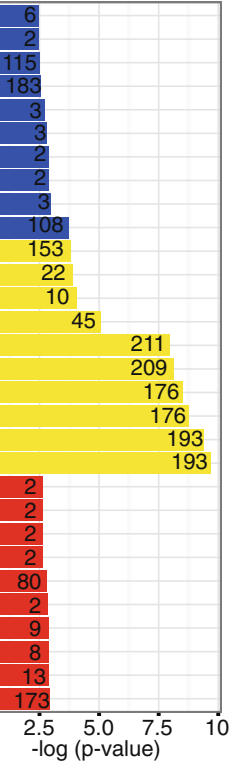

C

KEGG pathways

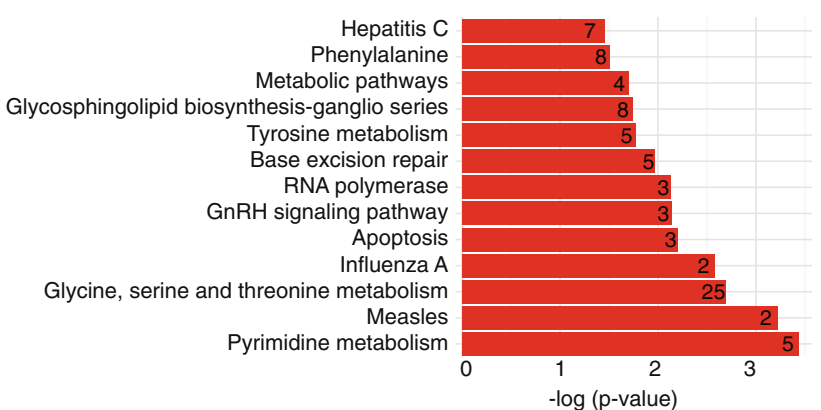

Fig. 7 Coexpression analysis of mRNAs, miRNAs and IncRNAs. a In total, 304 overlapped genes were found from mRNAs and targets of noncoding RNAs. $\mathbf{b}$ GO and $\mathbf{c}$ KEGG enrichment of overlapped genes (listed by $p$ value). Numbers in each row stand for the gene counts in each GO dataset or KEGG pathway. GO Gene Ontology, miRNA microRNA, IncRNA long noncoding RNA (Color figure online) 
Recent evidence suggests that adult pancreatic progenitors may provide a potential source for beta-cell neogenesis in adults [2]. In rodents, ductal epithelium has been proven to be a potential progenitor pool in vivo [7]. Partial duct ligation [7] or treatment with exendin-4 [8] induced ductal cell proliferation and differentiation into beta cells, suggesting that insulinproducing cells can be generated from adult pancreatic ductal cells. Just like embryonic pancreatic progenitors, in-vitro cultured murine adult pancreatic ductal cells can reacquire self-renewal capacity and pluripotency $[3,11-13,29]$. These cultured ductal cells can differentiate into insulin-producing cells in vivo [10] and in vitro $[9,29]$. Altogether, the adult pancreatic progenitors can serve as a promising beta-cell source for transplantation, although more research is needed.

Previously we reported that a 3D system was found to culture $\mathrm{CD} 133^{+} / \mathrm{SOX} 9^{\text {high }}$ pancreatic progenitor-like colonies [11]. This subpopulation could be expanded for many passages without losing its potential to differentiate to endocrine/acinar lineages. In addition, these cells can response to Wnt signal agonist R-spondin1. However, even if this is an excellent system to enrich these colonies, it is not perfect to study the regulation because of the conditioned medium which has uncertain components. Also, $\mathrm{CD} 133^{+} / \mathrm{SOX} 9^{\text {high }}$ were not proved to be all colony-forming units. To elucidate the mechanisms underlying the regulation of pancreatic progenitor cells, therefore, we modified the culturing system and used single cells from whole pancreas to generate pancreatic progenitor-like colonies and performed HTS to profile the transcriptome.

In our study, mouse pancreas was dissolved into single cells. In our modified system, these cells formed a cystlike organoid. These colonies were $\mathrm{PDX}^{+}, \mathrm{CK}^{+}, \mathrm{SOX}^{+}$ and weakly expressed NEUROG3. Also, they can differentiate to C-peptide secreting cells spontaneously. Following multiple passages, the cells did not lose self-renewal capacity and the ability to differentiate into other pancreatic lineages in a 2D system. Thus, our cultured pancreatic ductal epithelium exhibited pancreatic progenitor-like property and function.

To identify factors that regulate colony proliferation and differentiation, we profiled the colony cells' transcriptome using HTS, a widely used technology to study the gene expression profile [45]. Total RNAs including mRNAs, miRNAs and lncRNAs are sequenced and annotated. As well as RNAs that have been discovered, new RNAs (mainly miRNAs and lncRNAs) can also be sequenced using this technology. By comparing the RNA expression profile between two samples, many candidate genes of differential expression can be revealed. In this study, total RNA of the colonies was extracted and sequenced, using whole pancreas as control. We captured 7266 significantly differentially expressed mRNAs along with 285 miRNAs and 183 lncRNAs. Expression of a subset of identified genes was confirmed by qPCR to verify HTS reliability. We found that CD133 was enriched in the colonies (Fig. 4b) and only $\mathrm{CD} 133^{+}$cells could form colonies (Fig. 5b). We also found that most of the cells in ring colonies were $\mathrm{CD}_{133^{+}}$(Fig. 5a) and had a similar gene expression pattern to $\mathrm{CD}_{133^{+}}$cell-derived colonies (Fig. 5c). Because $\mathrm{CD}_{133^{+}}$cells should represent all of the cells with the ability to form pancreatic progenitor-like colonies, this implied that our colonies were proper materials for HTS.

In addition, we noted that Mettl10 is the most changed gene in upregulated mRNAs. METTL10 is a methyltransferase-like protein that trimethylates eukaryotic translation elongation factor 1 alpha 1(EF1A1), a eukaryotic elongation factor, which implies its contribution to many biological processes on a translational level [30]. This finding suggests that epigenetic regulation might play an important role in pancreatic progenitors. In order to find new surface markers, we verified quite a few cell surface proteins including signal pathway receptors, cell adhesion molecules and clusters of differentiation (CDs).

The miRNA showed an important role in beta-cell development [39]. A previous study showed that miR-21 regulates beta-cell death by targeting the tumorsuppressing gene $P d c d 4$ [46]. Nevertheless, little is known of whether miRNA plays important roles in regulating pancreatic progenitors. In our results, miR-21a, miR-31, miR-200c and miR-155 were upregulated and miR-217, miR-802, miR-375 and miR-216 were downregulated (Additional file 9: Table S5). One differentially expressed miRNA of particular interest is miR-802, a transcriptional factor highly expressed in the liver and pancreas that targets HNF1B to regulate glycometabolism [38]. In addition, HNF1B is a crucial factor in pancreas development [47] and loss of HNF1B exhibits pancreas hypoplasia [48]. Also, HNF1B is upstream of SOX9 and NEUROG3 $[48,49]$, indicating its latent function to regulate pancreatic progenitors. In our study, $H N F 1 B$ is upregulated in the colonies shown by HTS $\left(\log _{2}=4.55, p<0.05\right)$. Thus, miR-802 might be a potential miRNA regulating pancreatic progenitors. Another miRNA, miR-375, is a well-characterized miRNA regulating pancreas development [39]. miR-375 knockdown lead to reduced endocrine cells [50]. In conclusion, our profiling of the miRNA transcriptome provided a vast miRNA candidate pool for further research.

Our study also identified several differentially expressed IncRNAs, a group of noncoding RNAs which may regulate gene expression through various mechanisms [25]. Several IncRNAs play a role in pancreatic cancer [26], beta-cell 
biology [27] and pancreas development [28]. Recently a lncRNA was shown to regulate specification and function of beta cells by targeting multiple transcriptional factors [51]. Our profile identified 183 differentially expressed lncRNAs. In most upregulated lncRNAs, Malat1 was shown to promote pancreatic cancer proliferation by stimulating autophagy [41], indicating its regulation effect on cell proliferation.

To reveal the functions of differentially expressed RNAs in our colonies, GO and KEGG analyses were performed. We note that the term "metabolic" was enriched in the biological process of both mRNAs and miRNAs. Also, in KEGG analysis of mRNA, the metabolic pathway was found to be significant. Metabolic pathways have been shown to have an important role in regulating stem cell functions [52]. Stemness is regulated by metabolic pathways in stem cells [53]. It is likely that pancreatic progenitors may share the same metabolic condition with other kinds of stem/progenitor cells. Regulation of metabolic pathways may also affect their functions.

\section{Conclusion}

In summary, our study provides a system to enrich and culture pancreatic progenitor-like colonies in vitro, and HTS of the colonies should help understand the regulation of their proliferation and differentiation at a transcriptional level. HTS also offers a lot of candidate genes (including noncoding RNAs) which could be potential regulators or markers of pancreatic progenitors. By controlling these targets, we may manage to accumulate more colonies or force them to differentiate to beta lineage. In general, our results offer an efficient culture system for pancreatic progenitor-like colonies and the HTS of the colonies serves as a target resource for further study of in-vitro cultured pancreatic progenitors.

\section{Additional files}

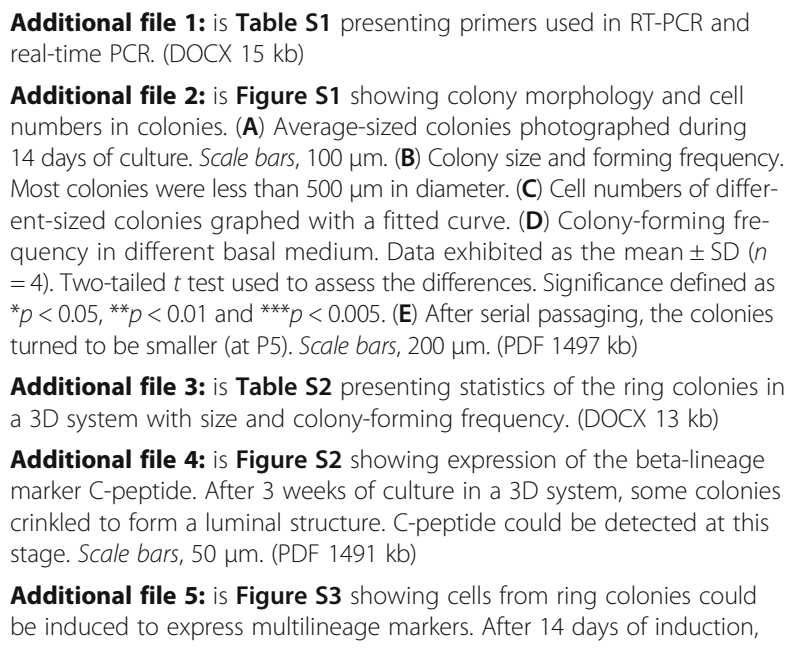

Additional file 5: is Figure S3 showing cells from ring colonies could be induced to express multilineage markers. After 14 days of induction,

RT-PCR confirmed the expression of Ins 1 , Ins 2 and other endocrine genes in clusters. Exocrine gene Amylase could also be detected in some clusters. (PDF $233 \mathrm{~kb}$ )

Additional file 6: is Figure $\mathbf{S 4}$ showing differentially expressed IncRNAs detected by HTS and verified by real-time PCR. (A) Heat map of all IncRNA of the colonies and control. (B) Verification of differentially expressed IncRNAs. Data exhibited as the mean \pm SD $(n=4)$. Two-tailed $t$ test was used to assess the differences. Significance defined as ${ }^{*} p<0.05,{ }^{* *} p<0.01$ and ${ }^{* * *} p<0.005$, comparison to the control group. (PDF $214 \mathrm{~kb}$ )

Additional file 7: is Table S3 presenting the number of significantly changed RNAs between colonies and control. (DOCX $12 \mathrm{~kb}$ )

Additional file 8: is Table S4 presenting top 25 differentially expressed mRNAs between colonies and control. (DOCX 14 kb)

Additional file 9: is Table S5 presenting top 25 differentially expressed miRNAs between colonies and control. (DOCX $14 \mathrm{~kb}$ )

Additional file 10: is Table S6 presenting top 25 differentially expressed IncRNAs between colonies and control. (DOCX 14 kb)

Additional file 11: is Table S7 presenting original HTS data sets for miRNAs. (XLSX $172 \mathrm{~kb}$ )

Additional file 12: is Table S8 presenting original HTS data sets for mRNAs and IncRNAs. (XLSX $12293 \mathrm{~kb}$ )

Additional file 13: is Figure S5 showing GO and KEGG enrichment of IncRNAs. (A) GO enrichment of significantly changed IncRNA targets. Top 10 (by $p$ value) of each GO dataset. Numbers in each part of the graph stand for the gene counts in each GO dataset. (B) Top 20 (by $p$ value) of enriched KEGG pathways of IncRNA targets. Numbers on top of each column stand for the gene count in each KEGG pathway. (PDF 83 kb)

Additional file 14: is Table S9 presenting top five overlapped genes and counts of targeting noncoding RNAs. (DOCX $13 \mathrm{~kb}$ )

\section{Abbreviations}

BSA: Bovine serum albumin; Cela1: Chymotrypsin-like elastase family, member 1; Ck7: Cytokeratin 7; Ctrl: Chymotrypsin; Cyc: Cyclophillin;

DIl1: Delta-like 1; EF1A1: Eukaryotic translation elongation factor 1 alpha 1; EGF: Epidermal growth factor; FBS: Fetal bovine serum; FCM: Flow cytometry; Fgfr2: Fibroblast growth factor receptor 2; Fzd1: Frizzled class receptor 1; GO: Gene Ontology; HNF1B: HNF1 homeobox B; HTS: High-throughput sequencing; Icam1: Intercellular adhesion molecule 1; KEGG: Kyoto Encyclopedia of Genes and Genomes; IncRNA: Long noncoding RNA; Mafa: v-maf musculoaponeurotic fibrosarcoma oncogene family, protein A: Malat1: Metastasis associated lung adenocarcinoma transcript 1; Mettl10: Methyltransferase like 10; miRNA: MicroRNA; Neurod1: Neurogenic differentiation 1; NEUROG3: Neurogenin 3; Nkx2.2: NK2 homeobox 2; Nkx6.1: NK6 homeobox 1; Pax4: Paired box 4; Pax6: Paired box 6; PCR: Polymerase chain reaction; Pdx1: Pancreatic and duodenal homeobox 1; Sox9: Sex determining region Y-box 9; TET: Ten-eleven translocation; Try4: Trypsin 4

\section{Acknowledgements}

Not applicable.

\section{Funding}

This project is funded by the National High Technology Research and Development Program of China (863 Program, No. 2015AA020314); supported by the National Natural Science Foundation of China (Grant No. 81570696 and No. 31270985); supported by the Excellent Youth Foundation of Jiangsu Scientific Committee (BK20140029); sponsored by the Qing Lan Project; and supported by the Program for Jiangsu Province Innovative Research Team, Priority Academic Program Development of Jiangsu Higher Education Institutions and Top-notch Academic Programs Project of Jiangsu Higher Education Institutions (PPZY2015A057).

\section{Availability of data and materials}

All data generated or analyzed during this study have been included in this published article and its supplementary information files. 


\section{Authors' contributions}

DM was responsible for performing research, data analysis, figure preparation, manuscript writing, and final approval of the manuscript. ST was responsible for performing animal experiments, data analysis, and final approval of the manuscript. JS, QW, FZ, YX, YP, and YZ were responsible for performing research and final approval of the manuscript. JJ, YZ, and $L J$ were responsible for conception and design, and final approval of manuscript. All authors read and approved the final manuscript.

\section{Ethics approva}

C57BL/6 J mice (6-8 weeks old) were obtained from the Model Animal Research Center of Nanjing University (Nanjing, China). All care and handling of animals were carried out according to the international laws and policies (EEC Council Directive 86/609, 1987) and approved by the animal ethics committee of China Pharmaceutical University (Nanjing, China) (Reference Number: 2162326)

\section{Consent for publication}

Not applicable.

\section{Competing interests}

The authors declare that they have no competing interests.

\section{Publisher's Note}

Springer Nature remains neutral with regard to jurisdictional claims in published maps and institutional affiliations.

Received: 27 February 2017 Revised: 16 June 2017

Accepted: 3 July 2017 Published online: 26 July 2017

\section{References}

1. Jiang FX, Morahan G. Pancreatic stem cells: from possible to probable. Stem Cell Rev. 2012;8(3):647-57.

2. Ramiya VK, Maraist M, Arfors KE, Schatz DA, Peck AB, Cornelius JG. Reversal of insulin-dependent diabetes using islets generated in vitro from pancreatic stem cells. Nat Med. 2000:6(3):278-82

3. Bonner-Weir S, Taneja M, Weir GC, Tatarkiewicz K, Song KH, Sharma A, et al. In vitro cultivation of human islets from expanded ductal tissue. Proc Nat Acad Sci U S A. 2000;97(14):7999-8004.

4. Rovira M, Scott SG, Liss AS, Jensen J, Thayer SP, Leach SD. Isolation and characterization of centroacinar/terminal ductal progenitor cells in adult mouse pancreas. Proc Natl Acad Sci U S A. 2010;107(1):75-80.

5. Minami K, Okuno M, Miyawaki K, Okumachi A, Ishizaki K, Oyama K, et al. Lineage tracing and characterization of insulin-secreting cells generated from adult pancreatic acinar cells. Proc Natl Acad Sci U S A. 2005:102(42):15116-21.

6. Corritore E, Lee YS, Sokal EM, Lysy PA. beta-cell replacement sources for type 1 diabetes: a focus on pancreatic ductal cells. Ther Adv Endocrinol Metab. 2016;7(4):182-99.

7. Bonner-Weir S, Toschi E, Inada A, Reitz P, Fonseca SY, Aye T, et al. The pancreatic ductal epithelium serves as a potential pool of progenitor cells. Pediatr Diabetes. 2004;5 Suppl 2:16-22.

8. Stoffers DA, Kieffer TJ, Hussain MA, Drucker DJ, Bonner-Weir S, Habener JF, et al. Insulinotropic glucagon-like peptide 1 agonists stimulate expression of homeodomain protein IDX-1 and increase islet size in mouse pancreas. Diabetes. 2000;49(5):741-8.

9. Corritore E, Dugnani E, Pasquale V, Misawa R, Witkowski P, Lei J, et al. Betacell differentiation of human pancreatic duct-derived cells after in vitro expansion. Cell Reprogram. 2014;16(6):456-66.

10. Huch M, Bonfanti P, Boj SF, Sato T, Loomans CJ, van de Wetering M, et al. Unlimited in vitro expansion of adult bi-potent pancreas progenitors through the Lgr5/R-spondin axis. Embo J. 2013;32(20):2708-21.

11. Jin L, Feng T, Shih HP, Zerda R, Luo A, Hsu J, et al. Colony-forming cells in the adult mouse pancreas are expandable in Matrigel and form endocrine/acinar colonies in laminin hydrogel. Proc Natl Acad Sci U S A. 2013;110(10):3907-12.

12. Oshima Y, Suzuki A, Kawashimo K, Ishikawa M, Ohkohchi N, Taniguchi H. Isolation of mouse pancreatic ductal progenitor cells expressing CD133 and c-Met by flow cytometric cell sorting. Gastroenterology. 2007;132(2):720-32.

13. Jin L, Feng T, Zerda R, Chen CC, Riggs AD, Ku HT. In vitro multilineage differentiation and self-renewal of single pancreatic colony-forming cells from adult C57BL/6 mice. Stem Cells Dev. 2014;23(8):899-909.
14. Carpino G, Renzi A, Cardinale V, Franchitto A, Onori P, Overi D, et al Progenitor cell niches in the human pancreatic duct system and associated pancreatic duct glands: an anatomical and immunophenotyping study. J Anat. 2016:228(3):474-86.

15. Yokoi N, Serikawa T, Walther R. Pdx1, a homeodomain transcription factor required for pancreas development, maps to rat chromosome 12. Exp Anim. 1997:46(4):323-4.

16. Boo L, Ho WY, Ali NM, Yeap SK, Ky H, Chan KG, et al. MiRNA Transcriptome profiling of spheroid-enriched cells with cancer stem cell properties in human breast MCF-7 cell line. Int J Biol Sci. 2016;12(4):427-45.

17. Casado-Diaz A, Anter J, Muller S, Winter P, Quesada-Gomez JM, Dorado G Transcriptomic analyses of adipocyte differentiation from human mesenchymal stromal-cells (MSC). J Cell Physiol. 2017;232(4):771-84.

18. Fogel GB, Kai ZS, Zargar S, Hinton A, Jones GA, Wong AS, et al. MicroRNA dynamics during human embryonic stem cell differentiation to pancreatic endoderm. Gene. 2015;574(2):359-70.

19. Hori Y. Prominin-1 (CD133) Reveals new faces of pancreatic progenitor cells and cancer stem cells: current knowledge and therapeutic perspectives. Adv Exp Med Biol. 2013;777:185-96.

20. Jin L, Gao D, Feng T, Tremblay JR, Ghazalli N, Luo A, et al. Cells with surface expression of CD133highCD71low are enriched for tripotent colony-forming progenitor cells in the adult murine pancreas. Stem Cell Res. 2016;16(1):40-53.

21. Bartel DP. MicroRNAs: genomics, biogenesis, mechanism, and function. Cell. 2004;116(2):281-97.

22. Francis N, Moore M, Rutter GA, Burns C. The role of microRNAs in the pancreatic differentiation of pluripotent stem cells. MicroRNA. 2014;3(1):54-63.

23. Filios SR, Shalev A. Beta-cell microRNAs: small but powerful. Diabetes. 2015; 64(11):3631-44

24. Fu $X$, Jin L, Wang $X$, Luo A, Hu J, Zheng $X$, et al. MicroRNA-26a targets ten eleven translocation enzymes and is regulated during pancreatic cell differentiation. Proc Natl Acad Sci U S A. 2013:110(44):17892-7.

25. Zhang D, Xiong M, Xu C, Xiang P, Zhong X. Long noncoding RNAs: an overview. Methods Mol Biol. 2016;1402:287-95.

26. Peng JF, Zhuang YY, Huang FT, Zhang SN. Noncoding RNAs and pancreatic cancer. World J Gastroenterol. 2016:22(2):801-14.

27. Singer RA, Arnes L, Sussel L. Noncoding RNAs in beta cell biology. Curr Opin Endocrinol Diabetes Obes. 2015;22(2):77-85.

28. Arnes L, Sussel L. Epigenetic modifications and long noncoding RNAs influence pancreas development and function. Trends Genet. 2015;31(6):290-9.

29. Gao R, Ustinov J, Pulkkinen MA, Lundin K, Korsgren O, Otonkoski T. Characterization of endocrine progenitor cells and critical factors for their differentiation in human adult pancreatic cell culture. Diabetes. 2003;52(8):2007-15

30. Shimazu T, Barjau J, Sohtome Y, Sodeoka M, Shinkai Y. Selenium-based Sadenosylmethionine analog reveals the mammalian seven-beta-strand methyltransferase METTL10 to be an EF1A1 lysine methyltransferase. PLoS One. 2014;9(8), e105394.

31. Klimova L, Antosova B, Kuzelova A, Strnad H, Kozmik Z. Onecut1 and Onecut2 transcription factors operate downstream of Pax6 to regulate horizontal cell development. Dev Biol. 2015;402(1):48-60.

32. Vanhorenbeeck V, Jenny M, Cornut JF, Gradwohl G, Lemaigre FP, Rousseau $\mathrm{GG}$, et al. Role of the Onecut transcription factors in pancreas morphogenesis and in pancreatic and enteric endocrine differentiation. Dev Biol. 2007:305(2):685-94.

33. Matsuda Y, Yoshimura H, Suzuki T, Uchida E, Naito Z, Ishiwata T. Inhibition of fibroblast growth factor receptor 2 attenuates proliferation and invasion of pancreatic cancer. Cancer Sci. 2014;105(9):1212-9.

34. Laurila EM, Sandstrom S, Rantanen LM, Autio R, Kallioniemi A. Both inhibition and enhanced expression of miR-31 lead to reduced migration and invasion of pancreatic cancer cells. Genes Chromosomes Cancer. 2012;51(6):557-68.

35. Bai C, Li X, Gao Y, Wang K, Fan Y, Zhang S, et al. Role of microRNA-21 in the formation of insulin-producing cells from pancreatic progenitor cells. Biochim Biophys Acta. 2016;1859(2):280-93.

36. Ali S, Dubaybo H, Brand RE, Sarkar FH. Differential expression of microRNAs in tissues and plasma co-exists as a biomarker for pancreatic cancer. J Cancer Sci Ther. 2015;7(11):336-46.

37. AC Azevedo-Pouly, DS Sutaria, J Jiang, OA Elgamal, F Amari, D Allard, et al. miR-216 and miR-217 expression is reduced in transgenic mouse models of pancreatic adenocarcinoma, knockout of miR-216/miR-217 host gene is embryonic lethal. Funct Integr Genomics. 2017;17(2-3):203-12. 
38. Kornfeld JW, Baitzel C, Konner AC, Nicholls HT, Vogt MC, Herrmanns K, et al. Obesity-induced overexpression of miR-802 impairs glucose metabolism through silencing of Hnf1b. Nature. 2013;494(7435):111-5.

39. M Kaviani, N Azarpira, MH Karimi, I Al-Abdullah. The role of microRNAs in islet beta-cell development. Cell Biol Int. 2016;40(12):1248-55.

40. Lahmy R, Soleimani M, Sanati MH, Behmanesh M, Kouhkan F, Mobarra N. MiRNA-375 promotes beta pancreatic differentiation in human induced pluripotent stem (hiPS) cells. Mol Biol Rep. 2014;41(4):2055-66.

41. Li L, Chen H, Gao Y, Wang YW, Zhang GQ, Pan SH, et al. Long noncoding RNA MALAT1 promotes aggressive pancreatic cancer proliferation and metastasis via the stimulation of autophagy. Mol Cancer Ther. 2016;15(9):2232-43.

42. Wilcox NS, Rui J, Hebrok M, Herold KC. Life and death of beta cells in type 1 diabetes: a comprehensive review. J Autoimmun. 2016;71:51-8.

43. Yagihashi S, Inaba W, Mizukami H. Dynamic pathology of islet endocrine cells in type 2 diabetes: beta-cell growth, death, regeneration and their clinical implications. J Diabetes Investig. 2016;7(2):155-65.

44. luamoto LR, Franco AS, Suguita FY, Essu FF, Oliveira LT, Kato JM, et al. Human islet xenotransplantation in rodents: a literature review of experimental model trends. Clinics (Sao Paulo). 2017;72(4):238-43.

45. Blodgett DM, Cura AJ, Harlan DM. The pancreatic beta-cell transcriptome and integrated-omics. Curr Opin Endocrinol Diabetes Obes. 2014;21(2):83-8.

46. Ruan Q, Wang T, Kameswaran V, Wei Q, Johnson DS, Matschinsky F, et al. The microRNA-21-PDCD4 axis prevents type 1 diabetes by blocking pancreatic beta cell death. Proc Natl Acad Sci U S A. 2011;108(29):12030-5.

47. Maestro MA, Cardalda C, Boj SF, Luco RF, Servitja JM, Ferrer J. Distinct roles of HNF1 beta, HNF1alpha, and HNF4alpha in regulating pancreas development, beta-cell function and growth. Endocr Dev. 2007;12:33-45.

48. De Vas MG, Kopp JL, Heliot C, Sander M, Cereghini S, Haumaitre C. Hnf1 b controls pancreas morphogenesis and the generation of $\mathrm{Ngn} 3+$ endocrine progenitors. Development. 2015;142(5):871-82

49. Yang Y, Chang BH, Yechoor V, Chen W, Li L, Tsai MJ, et al. The Kruppel-like zinc finger protein GLIS3 transactivates neurogenin 3 for proper fetal pancreatic islet differentiation in mice. Diabetologia. 2011;54(10):2595-605.

50. Kloosterman WP, Lagendijk AK, Ketting RF, Moulton JD, Plasterk RH. Targeted inhibition of miRNA maturation with morpholinos reveals a role for miR-375 in pancreatic islet development. PLoS Biol. 2007:5(8), e203.

51. Arnes L, Akerman I, Balderes DA, Ferrer J, Sussel L. betalincl encodes a long noncoding RNA that regulates islet beta-cell formation and function. Genes Dev. 2016;30(5):502-7.

52. Mlody B, Prigione A. A glycolytic solution for pluripotent stem cells. Cell Stem Cell. 2016;19(4):419-20.

53. Shafie A, Xue M, Barker G, Zehnder D, Thornalley PJ, Rabbani N. Reappraisal of putative glyoxalase 1-deficient mouse and dicarbonyl stress on embryonic stem cells in vitro. Biochem J. 2016:473(22):4255-70.

\section{Submit your next manuscript to BioMed Central and we will help you at every step:}

- We accept pre-submission inquiries

- Our selector tool helps you to find the most relevant journal

- We provide round the clock customer support

- Convenient online submission

- Thorough peer review

- Inclusion in PubMed and all major indexing services

- Maximum visibility for your research

Submit your manuscript at www.biomedcentral.com/submit

) Biomed Central 